\title{
Relationship between maternal blood ceruloplasmin level, catalase and myeloperoxidase activity and neural tube defects
}

\author{
Orkun Cetin ${ }^{1}$, Erbil Karaman ${ }^{1}$, Baris Boza ${ }^{1}$, Numan Cim ${ }^{1}$, Murat Alisik², Ozcan Erel2, \\ İsmet Alkis ${ }^{1}$, Recep Yildizhan ${ }^{1}$, Ali Kolusarı ${ }^{1}$, Guler Hanım Sahin ${ }^{1}$ \\ ${ }^{1}$ Yuzuncu Yil University, Faculty of Medicine, Department of Obstetrics and Gynecology, Van, Turkey \\ ${ }^{2}$ Yildirim Beyazid University, Faculty of Medicine, Department of Biochemistry, Ankara, Turkey
}

\begin{abstract}
Objectives: The exact pathogenesis of neural tube defects (NTDs) is poorly understood. We aimed at evaluating maternal anti-oxidant capacity (ceruloplasmin level, myeloperoxidase and catalase activity) in pregnancies complicated by NTDs.

Material and methods: Fifty-four mothers with NTD-affected pregnancies and 61 healthy mothers, matched for gestational age, were recruited. Maternal venous blood samples were obtained after detailed fetal ultrasound examination to measure myeloperoxidase, catalase activity and ceruloplasmin levels. The clinical characteristics of all participants were collected.

Results: Maternal blood catalase activity was significantly lower in the study group (117.1 $\pm 64.8 \mathrm{kU} / \mathrm{L})$ as compared to controls $(152.2 \pm 110.6 \mathrm{kU} / \mathrm{L})(p=0.044)$. Maternal blood ceruloplasmin levels were also significantly lower in the study group $(180.5 \pm 37.7 \mathrm{U} / \mathrm{L})$ as compared to controls $(197.9 \pm 35.9 \mathrm{U} / \mathrm{L})(\mathrm{p}=0.012)$. Myeloperoxidase activity was similar in both groups $(112.6 \pm 22.2 \mathrm{U} / \mathrm{L}$ vs. $113.6 \pm 38.1 \mathrm{U} / \mathrm{L})(\mathrm{p}=0.869)$.

Conclusions: In the present study, maternal blood ceruloplasmin level and catalase activity were found to be lower in NTD-affected pregnancies as compared to healthy controls. Thus, it seems safe to conclude that impaired antioxidant capacity may play a role in the development of NTDs during pregnancy, in addition to the genetic, environmental and metabolic factors.
\end{abstract} Key words: pregnancy, neural tube defects, catalase, myeloperoxidase, ceruloplasmin

Ginekologia Polska 2017; 88, 3: 156-160

\section{INTRODUCTION}

Neural tube defects (NTDs) are a group of heterogeneous and complex central nervous system disorders, affecting approximately $0.5-2 / 1000$ pregnancies [1], and contributing to significant fetal-neonatal morbidity and mortality. Most NTDs result from the primary failure of the embryonic neural tube closure during early embryogenesis. The well-known mechanisms for the development of NTDs include folate deficiency, in utero drug exposure, and genetic susceptibility [2]. However, the exact pathogenesis of NTDs remains to be fully elucidated [3]. It has been reported that the direct relationship between antiepileptic drug therapy and the development of NTD is attributed to the formation of toxic drug metabolites and free radical damage [4].
Oxidative metabolism was reported to be related with congenital anomalies, including NTDs [3]. Free radicals or reactive oxygen species are generated during aerobic respiration and metabolism. As a response, antioxidant defense mechanisms - including enzymes and chemicals which prevent free radical-induced damages to lipids, proteins and DNA - are produced by the human cells [5]. Ceruloplasmin is an acute-phase response protein which transports copper to the tissue sites and acts as an antioxidant which functions as an extracellular scavenger of free radicals and superoxide ions [6]. Free radicals play a significant role in the development of meningomyelocele (MM), and there is a direct relationship between antioxidant enzymes, including catalase (CAT) and myeloperoxidase (MPO), and the development 
of NTD [7]. CAT is an intracellular antioxidant enzyme and its production increases during oxidative stress in order to balance redox reactions [5]. MPO is another intracellular antioxidant enzyme which is stored in neutrophil granules. An increase in the production of free radicals or reduction in these antioxidant systems will result in oxidative stress, which is found to be related to numerous disorders, including inflammation, infection, diabetes, cancer, and cardiovascular disease. Recent reports have associated oxidative stress with congenital anomalies, including NTDs $[3,8]$.

\section{OBJECTIVES}

In this study, maternal blood ceruloplasmin level, catalase and MPO activities in pregnancies complicated by NTDs were investigated and compared to pregnant women with healthy fetuses.

\section{MATERIAL AND METHODS}

The present study was conducted at Yuzuncu YII University Medical Faculty, Department of Obstetrics and Gynecology, between January 2015 and July 2015. Local Ethics Committee approved of the study and informed consent was obtained from all participants. The principles of the Declaration of Helsinki were followed. The study population included 122 pregnant women, aged 18-45 years, who were investigated at our referral obstetric center. All subjects were followed-up until delivery. NTDs were not linked to genetic syndromes, amniotic band disruption sequence, or aneuploidies. Healthy pregnant women had no earlier history of congenital malformations. During the postnatal period, the diagnosis of NTDs was made by a pediatrician and further confirmed by a neurosurgeon. The exclusion criteria were as follows: fetal anomalies outside the central nervous system, e.g. esophageal atresia, congenital heart or renal disease; multiple pregnancy, chronic maternal disease, e.g. heart disease or diabetes, and alcohol or smoke addiction, delivery in the study group and lack of NTDs confirmation during the postnatal period. All in all, 54 pregnant women affected by NTDs who fulfilled the inclusion criteria comprised the study group. Seven pregnant women with additional fetal congenital anomalies were excluded. The control group consisted of pregnant women, matched for gestational age, who were referred to our clinic for detailed fetal ultrasound examination and diagnosed with a healthy fetus. After detailed ultrasound examination, maternal serum samples were obtained and stored until assayed.

The study group included 54 mothers with NTD-affected pregnancies and the control group consisted of 61 mothers with healthy pregnancies, matched for gestational age. The NTD group comprised of 18 fetuses with anencephaly (18/54, 33.3\%), 9 with thoracolumbar spina bifida (9/54, $16.7 \%), 20$ with lumbosacral spina bifida (20/54, 37\%), and 7 with encephalocele (7/54, 13\%). Patient characteristics (maternal age, gravidity, parity, BMI and gestational age at sampling) were recorded.

Fasting venous blood samples were drawn after diagnostic ultrasonography examination to evaluate MPO, ceruloplasmin and catalase levels. Blood samples were centrifuged quickly, sera were separated after centrifugation at $1500 \mathrm{rpm}$ for $10 \mathrm{~min}$, and stored at $-80^{\circ} \mathrm{C}$ until delivery.

Catalase activity was measured using the Goth's method [9]. The sample $(0.2 \mathrm{~mL})$ was incubated in $1.0 \mathrm{~mL}$ substrate $\left(65 \mathrm{mmol}\right.$ per $\mathrm{H}_{2} \mathrm{O}_{2}$ in $60 \mathrm{mmol} / \mathrm{L}$ sodium-potassium phosphate buffer, $\mathrm{pH} 7.4$ ) at $37^{\circ} \mathrm{C}$ for $60 \mathrm{~s}$. The enzymatic reaction was stopped with $1.0 \mathrm{~mL}$ of $32.4 \mathrm{Mm}$ ammonium molybdate, and the yellow complex of molybdate and $\mathrm{H}_{2} \mathrm{O}_{2}$ was measured at $405 \mathrm{~nm}$ against a blank. One unit of catalase decomposes $1 \mu \mathrm{mol}$ of $\mathrm{H}_{2} \mathrm{O}_{2} \mathrm{~min}^{-1}$ under these conditions. The results were expressed as kU/L. Serum MPO activity was measured by modification of the o-Dianisidine method [10] based on kinetic measurement at $460 \mathrm{~nm}$, with the rate of the yellowish orange product formation from the oxidation of o-Dianisidine with MPO in the presence of hydrogen peroxide $\left(\mathrm{H}_{2} \mathrm{O}_{2}\right)$. One unit of MPO was defined as that degrading $1 \mu \mathrm{mol}$ of $\mathrm{H}_{2} \mathrm{O}_{2}$ min- 1 at $25^{\circ} \mathrm{C}$. MPO activity was expressed in units per liter serum. Ceruloplasmin levels were measured by the method described by Erel [11]. This method is automated, colorimetric, and based on the enzymatic oxidation of ferrous ion to ferric ion. The results were expressed in units per liter serum.

Descriptive statistics for continuous variables (characteristics) were presented as mean and standard deviation. Student's t-test was used to compare the continuous variables between the control and study groups. PASTE programs were used to analyze the data. Statistical significance level was considered as 5\%. SPSS (IBM Corp. Released 2013. IBM SPSS Statistics for Windows, Version 22.0. Armonk, NY) was used for all statistical computations.

\section{RESULTS}

A total of 115 pregnant women were included, divided into two groups: 54 mothers with NTD-affected pregnancies (study group) and 61 healthy, matched for gestational age, mothers (control group). The clinical and laboratory characteristics of both groups were presented in Table 1. Mean maternal age was $27.4 \pm 5.8$ years in the study population and did not differ between the study and control groups ( $p=0.691$ ). Mean gestational age was $20.8 \pm 4.8$ weeks in the study population. Mean gestational age at sampling did not differ between the study and control groups ( $p=0.897)$. No significant differences between the groups in terms of gravidity, parity and BMI were observed.

Maternal blood catalase activity was significantly lower in the study group $(117.1 \pm 64.8 \mathrm{kU} / \mathrm{L})$ as compared to con- 
Table 1. The baseline clinical characteristics of the participants

\begin{tabular}{|l|c|c|c|}
\hline & Controls $(\mathbf{n}=\mathbf{6 1})$ & Cases $(\mathbf{n}=\mathbf{5 4})$ & $\mathbf{p}^{*}$ \\
\hline Maternal age (years) & $27.2 \pm 5.5$ & $27.6 \pm 6.2$ & 0.691 \\
\hline Gravidity & $2.6 \pm 1.5$ & $2.9 \pm 2.0$ & 0.330 \\
\hline Parity & $1.4 \pm 1.4$ & $1.7 \pm 1.8$ & 0.368 \\
\hline BMI $\left[\mathrm{kg} / \mathrm{m}^{2}\right]$ & $25.7 \pm 3.6$ & $25.5 \pm 3.0$ & 0.801 \\
\hline Gestational age at sampling (weeks) & $20.8 \pm 2.4$ & $20.9 \pm 6.6$ & 0.897 \\
\hline Catalase $[\mathrm{kU} / \mathrm{L}]$ & $152.2 \pm 110.6$ & $117.1 \pm 64.8$ & 0.044 \\
\hline Ceruloplasmin [U/L] & $197.9 \pm 35.9$ & $180.5 \pm 37.7$ & 0.012 \\
\hline MPO [U/L] & $113.6 \pm 38.1$ & $112.6 \pm 22.2$ & 0.869 \\
\hline
\end{tabular}

*Students T-test was used for continuous variables

Cases - mothers with neural tube defect-affected pregnancies; controls - healthy mothers with unaffected pregnancies; BMI — body mass index; $\mathrm{MPO}$ - myeloperoxidase

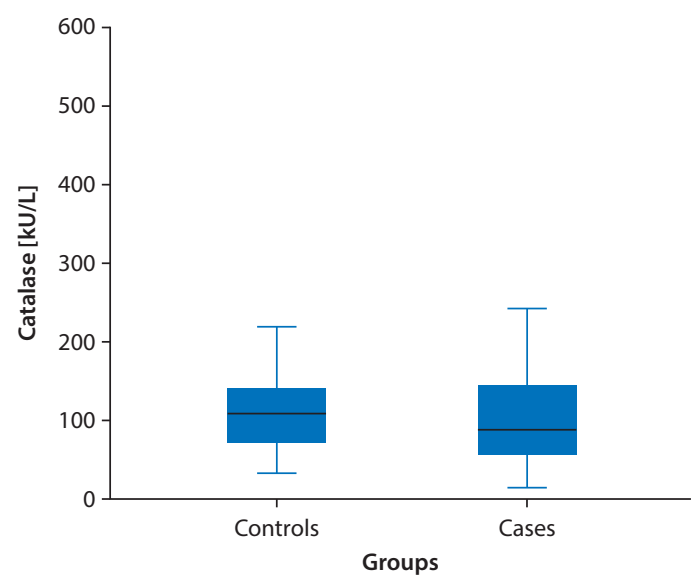

Figure 1. Maternal blood catalase levels of the study and control groups

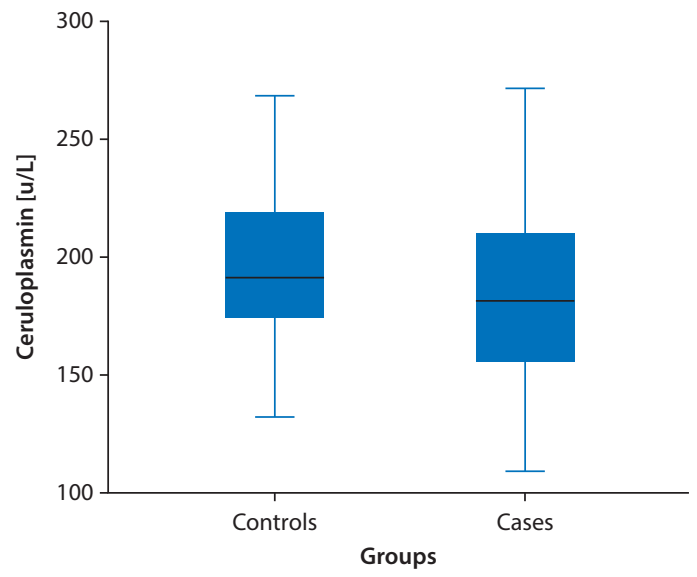

Figure 2. Maternal blood ceruloplasmin levels of the study and control groups

trols (152.2 $\pm 110.6 \mathrm{kU} / \mathrm{L})(\mathrm{p}=0.044)$ (Fig. 1). Maternal blood ceruloplasmin levels were also significantly lower in the study group $(180.5 \pm 37.7 \mathrm{U} / \mathrm{L})$ as compared to controls

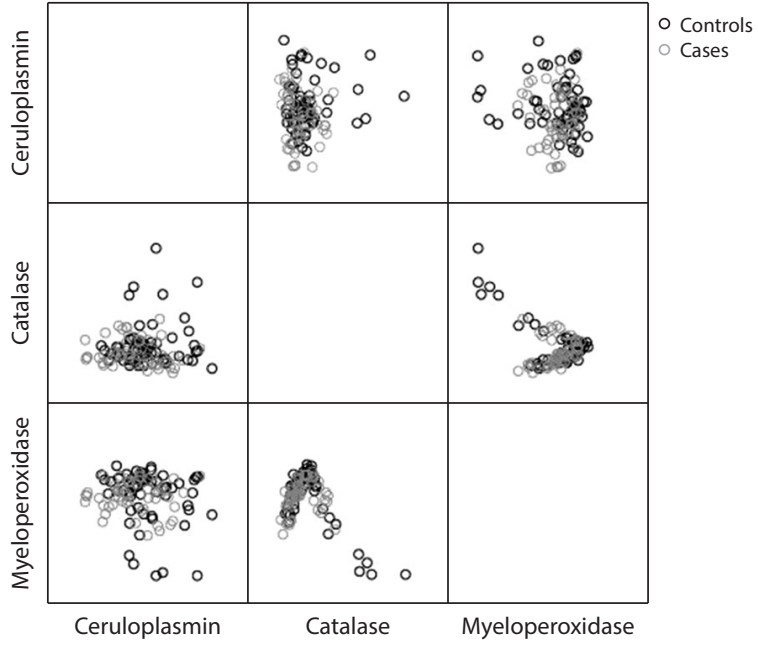

Figure 3. The scatter chart of catalase, MPO activity and ceruloplasmin levels between the study and control groups

$(197.9 \pm 35.9 \mathrm{U} / \mathrm{L})(\mathrm{p}=0.012)$ (Fig. 2). MPO activity was similar in both groups $(112.6 \pm 22.2 \mathrm{U} / \mathrm{L}$ vs. $113.6 \pm 38.1 \mathrm{U} / \mathrm{L})$ $(p=0.869)$. Figure 3 demonstrated the scatter chart of ceruloplasmin, catalase and MPO levels in the study and control groups.

\section{DISCUSSION}

In the present study, maternal blood levels of ceruloplasmin and catalase activity were found to be lower in pregnant women with NTD-complicated pregnancies as compared to healthy controls. However, MPO activity was similar in both groups. Although the exact etiology of the development of NTDs remains unknown, the neural tube closure during early embryogenesis can be affected by several factors, including metabolic, genetic and environmental conditions. Several studies have demonstrated a relationship between free oxygen species and NTDs [12,13]. The well-established data on free-radical mediated cellular damage comes from the 
use of antiepileptic drug complicated with NTDs [7]. In their experimental rat study, Ishibasia et al. showed that exposure to high oxygen concentration during early neurulation, aggravating oxidative stress in the embryo, leads to an increase in NTD incidence [14].

Reactive oxygen species (ROS) production is a natural process in the body and, given the idea that increased levels of ROS can cause damage to both, the human body and the developing fetus in utero [5], the body produces a variety of enzymatic and non-enzymatic mechanisms to protect the cells against oxidative stress. These include catalase, superoxide dismutase, MPO, glutathione peroxidase, ferritin and also ceruloplasmin, which act as antioxidants by removing iron which induces the oxidative reactions. It has been reported that impaired responsiveness of these antioxidant enzymes to free radicals and oxidative stress plays a significant role in the oxygen-induced embryopathy and might result in NTDs [15]. Catalase and MPO are known as intracellular antioxidant enzymes and catalase is found primarily in peroxisomes; it catalyzes a reaction between two $\mathrm{H}_{2} \mathrm{O}_{2}$ molecules, resulting in the formation of water and $\mathrm{O}_{2}$. In addition, catalase can promote the interaction of $\mathrm{H}_{2} \mathrm{O}_{2}$ with hydrogen donors so that $\mathrm{H}_{2} \mathrm{O}_{2}$ can be converted to one molecule of water, and the reduced donor becomes oxidized (peroxidatic activity of catalase) [9]. Also, ceruloplasmin acts as a potent antioxidant molecule by means of the oxidation of aromatic amines [8].

To date, there is only a limited number of studies which evaluate the oxidative status of pregnancies complicated with NTDs. Yazıcıoğlu et al. studied maternal blood of second trimester fetuses, with and without NTDs, and reported that ceruloplasmin levels of maternal blood were not different in the two groups. They concluded that maybe other antioxidants, but not ceruloplasmin, play a role in NTD development [6]. In contrast to that study, we added catalase and MPO as antioxidants, besides ceruloplasmin level, and found that catalase and ceruloplasmin were significantly lower in pregnancies complicated by NTD. On the other hand, Arslan et al. studied the role of erythrocyte free radical scavenging enzyme activities (FRSE), carbonic anhydrase (CA) activity and malondialdehyde (MDA) in infants with MM and their mothers, but not during pregnancy, and reported that FRSE activities were lower in all infants with MM and their mothers as compared to healthy infants and their mothers. Arslan et al. stated that lower FRSE activities may indicate an increased frequency of MM [16], which consistent with our findings of lower catalase and ceruloplasmin level in pregnancies with NTD.

Graf et al. evaluated five different antioxidant enzymes, including catalase, in children with MM and found significantly lower glutathione peroxidase activities [12]. They stat- ed that the contribution of oxidative stress to human birth defects warrants further investigation. However, their study was conducted in children with NTDs but not during the intrauterine period. We thought that to investigate the oxidative status of these children in utero, during the developmental period, would allow for a comprehensive presentation of data regarding the potential relationships between oxidative stress and energy metabolism during primary neurulation. In our study, we found lower maternal blood catalase activity and ceruloplasmin level at NTD diagnosis during pregnancy as compared to healthy pregnant women. It may show somehow a proof of inadequate antioxidant mechanisms protecting from oxidative stress under some circumstances, especially in terms of the development of congenital defects.

Several limitations of the present study should be considered. First, the activity and levels of antioxidant molecules other than catalase, MPO and ceruloplasmin remain to be examined. Perhaps other antioxidants play a role in the development of NTDs. Second, we did not analyze the antioxidant status of the pregnant women at the beginning of gestation, especially during the period of organogenesis. NTDs are very early-onset congenital anomalies; an investigation of the oxidative status during embryogenesis may give a more comprehensive data regarding the etiopathogenesis of this disorder. On the other hand, the main strength of our study is the relatively larger sample size. Also, to the best of our knowledge, there have been no studies on maternal blood catalase, MPO activities and ceruloplasmin level in pregnancies complicated by NTDs. Our study has been the first to evaluate these antioxidants during pregnancy complicated by NTDs.

\section{CONCLUSIONS}

Based on our findings, we concluded that the decreased catalase activity and ceruloplasmin level are associated with impaired antioxidant capacity, which may play a role in the development of NTDs in pregnancy, in addition to the genetic, environmental and metabolic factors. Although we have demonstrated slight differences in maternal ceruloplasmin and catalase levels between NTD-affected pregnancies and healthy controls, these results could not be used as parameters for prenatal NTD screening. Further studies, with well-structured and larger populations conducted at the early period of embryogenesis, are needed to investigate the matter further.

\section{Acknowledgements}

The current study was conducted at Yuzuncu Yıl University Medical Faculty, Department of Obstetrics and Gynecology between January 2015 and July 2015. 


\section{Conflict of interest}

The authors declare that they have no conflict of interest. The authors indicated that there was no financial relationship with the organization that sponsored the research. The authors had full control of all primary data and we agree to allow the Journal to review our data if requested.

\section{REFERENCES}

1. Copp AJ, Stanier P, Greene NDE. Neural tube defects: recent advances, unsolved questions, and controversies. Lancet Neurol. 2013; 12(8): 799-810, doi: 10.1016/S1474-4422(13)70110-8, indexed in Pubmed: 23790957.

2. Daly LE, Kirke PN, Molloy A, et al. Folate levels and neural tube defects. Implications for prevention. JAMA. 1995; 274(21): 1698-1702, indexed in Pubmed: 7474275.

3. Perrone $\mathrm{S}$, Longini M, Bellieni CV, et al. Early oxidative stress in amniotic fluid of pregnancies with Down syndrome. Clin Biochem. 2007; 40(3-4): 177-180, doi: 10.1016/j.clinbiochem.2006.10.019, indexed in Pubmed: 17208212.

4. Finnell RH. Teratology: general considerations and principles. J Allergy Clin Immunol. 1999; 103(2): S337-S342, indexed in Pubmed: 9949334.

5. Agarwal A, Gupta S, Sekhon L, et al. Redox considerations in female reproductive function and assisted reproduction: from molecular mechanisms to health implications. Antioxid Redox Signal. 2008; 10(8): 1375-1403, doi: 10.1089/ars.2007.1964, indexed in Pubmed: 18402550.

6. Yazıcıoğlu C, Cebesoy FB, Balat O, et al. The relationship of ceruloplasmin and neural tube defects. J Turk Ger Gynecol Assoc. 2010; 11(2): 86-88, doi: 10.5152/jtgga.2010.05, indexed in Pubmed: 24591905.
7. Pippenger CE. Pharmacology of neural tube defects. Epilepsia. 2003; 44 Suppl 3: 24-32, indexed in Pubmed: 12790883.

8. Uriu-Adams JY, Keen CL. Copper, oxidative stress, and human health. Mol Aspects Med. 2005; 26(4-5): 268-298, doi: 10.1016/j.mam.2005.07.015, indexed in Pubmed: 16112185.

9. Góth L. A simple method for determination of serum catalase activity and revision of reference range. Clin Chim Acta. 1991; 196(2-3): 143-151, indexed in Pubmed: 2029780.

10. Bradley PP, Priebat DA, Christensen RD, et al. Measurement of cutaneous inflammation: estimation of neutrophil content with an enzyme marker. J Invest Dermatol. 1982; 78(3): 206-209, indexed in Pubmed: 6276474.

11. Erel O. Automated measurement of serum ferroxidase activity. Clin Chem. 1998; 44(11): 2313-2319, indexed in Pubmed: 9799759.

12. Graf WD, Oleinik OE, Pippenger $C E$, et al. Comparison of erythrocyte antioxidant enzyme activities and embryologic level of neural tube defects. Eur J Pediatr Surg. 1995; 5 Suppl 1: 8-11, doi: 10.1055/s-2008-1066253, indexed in Pubmed: 8770569.

13. Zhao W, Mosley BS, Cleves MA, et al. Neural tube defects and maternal biomarkers of folate, homocysteine, and glutathione metabolism. Birth Defects Res A Clin Mol Teratol. 2006; 76(4): 230-236, doi: 10.1002/bdra.20240, indexed in Pubmed: 16575882.

14. Ishibashi M, Akazawa S, Sakamaki H, et al. Oxygen-induced embryopathy and the significance of glutathione-dependent antioxidant system in the rat embryo during early organogenesis. Free Radic Biol Med. 1997; 22(3): 447-454, indexed in Pubmed: 8981036.

15. Ornoy A. Embryonic oxidative stress as a mechanism of teratogenesis with special emphasis on diabetic embryopathy. Reprod Toxicol. 2007; 24(1): 31-41, doi: 10.1016/j.reprotox.2007.04.004, indexed in Pubmed: 17548185.

16. Arslan $M$, Melek $M$, Demir $H$, et al. Relationship of antioxidant enzyme activities with myelomeningocele. Turk Neurosurg. 2012; 22(3): 300-304, doi: 10.5137/1019-5149.JTN.5177-11.1, indexed in Pubmed: 22664996. 